\title{
FULLY-AUTOMATED DRIVING: THE ROAD TO FUTURE VEHICLES
}

\author{
Hamish Jamson, Natasha Merat, Oliver Carsten, \& Frank Lai \\ Institute for Transport Studies, University of Leeds \\ Leeds, UK \\ Email: a.h.jamson@its.leeds.ac.uk
}

\begin{abstract}
Summary: The study investigated the impact of fully-automated vehicle control on driver behaviour, physiology and the uptake of secondary tasks in varying traffic conditions. Previous studies have indicated the potential ironies of such automation on fatigue, stress and situational awareness, but have also suggested potential benefits through enhanced safety, more efficient traffic flows and reduced driver workload. The research was undertaken in a high-fidelity driving simulator that allowed drivers to see, feel and hear the impact of the automated control. Independent factors of Drive Type (manual control, fully-automated) and Traffic Density (light, heavy) were manipulated in a repeated-measures experimental design. 49 drivers participated. Drivers experiencing full vehicle automation tended to refrain from behaviours, such as overtaking, that required them to temporarily retake manual control, accepting the resulting increase in journey time. Automation improved safety margins in car following, but this benefit was restricted only to conditions of light surrounding traffic. Automation also reduced heart rate and increased driver fatigue, the latter being mitigated somewhat by high traffic density. Furthermore, drivers became more heavily involved with in-vehicle entertainment than they were in manual driving, affording less visual attention to the road ahead. Drivers do appear happy to forgo their supervisory responsibilities in preference of a more entertaining automated drive. However, these responsibilities are taken more seriously as supervisory demand increases.
\end{abstract}

\section{INTRODUCTION}

Advanced Driver Assistance Systems (ADAS) have the potential to improve road transport safety, efficiency and comfort, becoming a major focus for automobile manufacturers in emerging vehicle designs. Many ADAS supporting vehicle control currently exist, and recently much attention has been afforded on both sides of the Atlantic to the development and supporting infrastructure of a fully-automated, self-driving vehicle (e.g. US National Automated Highway System Research Program, EU ADASE series of integrated projects).

Parasuraman, Sheridan and Wickens (2000) proposed that automation can be introduced for any task at any of four information-processing stages: information acquisition, information analysis, decision making and action, with each stage having its own levels of automation. The highest level of stage 3 automation (decision making) defines the control undertaken by the highest level of stage 4 automation (action) without requiring, or even allowing, any human involvement. This is the context which characterises fully-automated driving: the driver is no longer actively involved in routine vehicle handling, taking on a purely supervisory role in ensuring that the vehicle suitably performs the required control actions on their behalf. Such systems have been 
the focus of research into driver-vehicle interaction for some time (e.g. Nilsson, 1995) and clearly have significant potential benefits, highlighted in a review by Stanton and Marsden (1996) as follows:

- Improved well-being through the reduction of driver stress.

- Enhanced safety through a reduction in error and a restriction of driving style, commonly associated with accidents due to lapses and violations. Indeed, consistent and predictable vehicle behaviour is one of the arguments in favour of cooperative vehicles for more efficient roadway usage.

- The freeing-up of attentional resources allowing the driver greater capacity to attend to secondary tasks whilst driving.

- Increased sales through a greater attractiveness and desirability for those vehicles demonstrating a technical superiority over rivals.

In general, such advantages transpire when vehicle automation makes the driving task easier to accomplish; however, this has not always been the experience in parallel domains. Ever since such technologies began to reduce the burden on aeroplane pilots by automating their flying task, the question has been not whether functions can be automated but whether they should be (e.g. Wiener and Curry, 1980). Such early concerns were also highlighted in Bainbridge's (1983) seminal paper on the ironies of automation. Two main ironies were postulated: inappropriate assumptions made by the designers of automated systems about their operators, and the challenge of the tasks that still remain for operators to perform even after automation. Hence, whilst automation should make the task easier, often it does not (see Norman, 2007 for a review). Such HMI concerns are equally applicable in the context of fully-automated driving.

This study was devised to investigate driver behaviour with and without full vehicle automation in varying traffic conditions, providing changing demands in terms of vehicle control and supervisory requirements. The rationale to vary simulated traffic conditions was twofold. First, it allowed a manipulation of vehicle-controller demand, be that the human driver or the automated system. Furthermore, it provided the opportunity to assess whether that increased demand was influential on driver physiology or behaviour. As a result, the specific research hypotheses which could be evaluated were that vehicle automation:

- permits more efficient traffic flow,

- creates a safer driving environment, with a reduction of time spent at short headways, and

- captivates a less stressful driving environment, freeing up opportunity and capacity for involvement in secondary tasks, unrelated to driving.

\section{METHOD}

\section{Apparatus}

The study was performed using the University of Leeds Driving Simulator (Figure 1). The Jaguar vehicle cab is housed within a $4 \mathrm{~m}$ diameter, spherical projection dome. Eight visual channels are rendered at 60 frames/s. The five forward channels, each at a resolution of $1024 \times 768$, are front-projected providing a horizontal field of view of $250^{\circ}$. The three rear channels can be seen through the vehicle's central view and side mirrors, the latter both 
physically modified to accommodate $960 \times 480$ LCD panels. The simulator also incorporates an eight degree-of-freedom electrical motion system consisting of a 500mm stroke-length electrical hexapod mounted on a railed gantry affording $5 \mathrm{~m}$ of extra surge and sway.
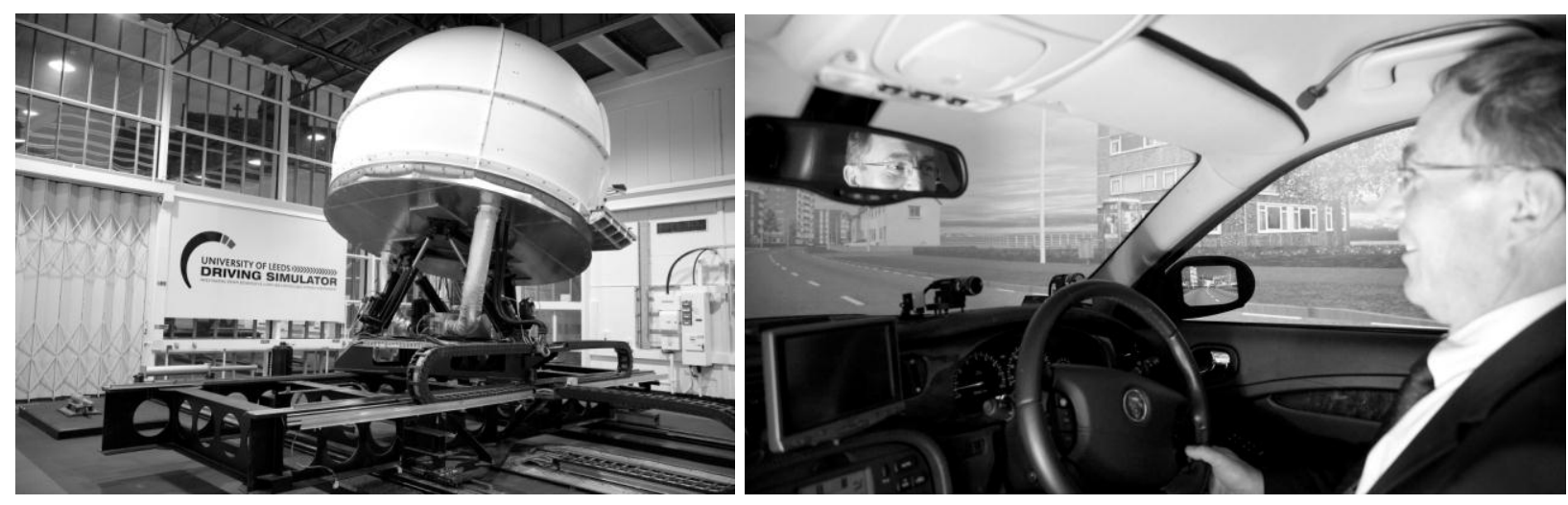

Figure 1. Exterior view (left) and vehicle cab (right) of the University of Leeds Driving Simulator

\section{Experimental design}

A two-factor, repeated-measures design was employed with the independent variables defined as Drive Type and Traffic Density.

Drive Type described the degree of automation available to the driver and had two levels: manual and fully-automated. In the manual driving condition, participants were entirely responsible for vehicle control. In the fully-automated condition, equivalent control inputs were made automatically by a pair of longitudinal (Ioannou, Xu, Eckert, Clemons and Sieja, 1993) and lateral (Sharp, Casanova and Symonds, 2000) controllers. The longitudinal controller was effectively an ACC with a 70mph default target speed and 1.5s headway. The lateral controller resembled a Lane Keeping System (LKS); its algorithm projected a series of look-ahead points in front of the vehicle before calculating the error from the desired trajectory. The resulting steer angle command attempted to maintain the vehicle in the centre of the lane occupied on activation of the LKS. Fully-automated driving was activated on request by depressing a built-in button mounted next the left-hand grip of the steering wheel. It was deactivated by either pushing the same button (toggle on/off), moving the steering wheel by more than $3^{\circ}$ from its current position or by depressing the brake pedal. A small LCD panel forming part of the speedometer was backlit to indicate the status of the fully-automated system (illuminated implied active).

The behaviour of the virtual traffic was manipulated to control Traffic Density at two levels: light and heavy. This was achieved by moving the location of the sources and sinks in the area of interest around the simulator vehicle and each agent's behavioural parameters. Light and heavy virtual traffic conditions were managed by the simulation to correspond to a lane count of 500 and 1500 vehicles per hour per lane respectively. The virtual driving scenario consisted throughout of a standard U.K. three-lane motorway. Data were collected over two 20.4 mile $(32.6 \mathrm{~km})$ sections of roadway, during which each level of Traffic Density existed. Between each section, a further 6.8 miles $(10.9 \mathrm{~km})$ allowed time for traffic conditions to be manipulated, unnoticeable to the participant; data were not recorded during this period. 


\section{Procedure}

Data were collected during a single session, lasting around two hours. On completion of informed consent, participants were familiarised with the simulator when driven manually and by the fully-automated system. Each condition of Drive Type was completed in a separate driving trial, counterbalanced, both taking around 45 minutes to complete and separated by a short break. Within each trial, drivers experienced both levels of Traffic Density, also counterbalanced. Use of the automated system was voluntary, but participants were briefed to "hand control over or back to the car as soon as you are comfortable". Drivers were told to imagine that they were on an unrushed journey and given the junction to exit the motorway.

In order to investigate the tendency for secondary activity uptake (i.e. those unrelated to driving), participants were provided with a range of in-vehicle entertainment, including a choice of films and TV programmes that could be viewed via a DVD player located in the vehicle and displayed on a LCD panel mounted in the central console of the dashboard. Use of the vehicle radio was also permitted. Participants were informed that they were free to engage in any activities at will, if and when they felt it was appropriate to do so. Involvement in each activity was logged manually by the researcher viewing remotely from the simulator's control room. Logging, equating to the duration that a task was adopted, was networked to the main simulation so that activities could be matched with heart rate, eye-tracking and recorded driving behaviour.

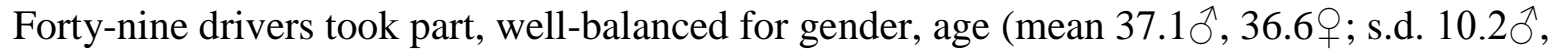
7.4ㅇ), driver experience (mean years licensed 17.7 $\hat{\jmath}, 17.4 \circ$; s.d. 11.0 $\hat{\jmath}, 7.3 \uparrow$ ) and annual

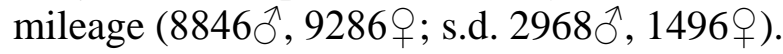

\section{RESULTS}

\section{Driver behaviour}

Figure 2 shows the proportion of time spent in each of the three available driving. In heavy traffic, participants logically tended to move towards the right lanes in order to overtake slower moving traffic (U.K. driving). With full vehicle automation, however, there was much less of a predilection to change lane. Instead participants generally appeared content let the system takes its course, showing a lack of desire to disengage and retake manual control for an overtaking manoeuvre: increase in lane 2 occupation from manual $(49.5 \%)$ to automated $(71.5 \%)$; $\mathrm{F}(1,48)=24.1, p<.001, \eta^{2}=.34$; decrease in lane 3 occupation from manual $(32.3 \%)$ to automated $(13.3 \%) ; \mathrm{F}(1,48)=27.3, p<.001, \eta^{2}=.36$. There was also a significant interaction of Drive Type and Traffic Density for lane 3 occupation; $\mathrm{F}(1,48)=5.77, p=.020, \eta^{2}=.11$. When experiencing fully-automated control, drivers spent a significantly smaller proportion of their journey overtaking in lane 3 , even in heavy traffic conditions.

The tendency not to overtake with automation significantly increased the time to complete a road section and reduced the mean speed achieved. Journey time increased from manual (1029s) to fully-automated (1101s), equivalent to a reduction in mean speed from manual $(72.0 \mathrm{mph})$ to fully-automated (66.9 mph); $\mathrm{F}(1,48)=50.2, p<.001, \eta^{2}=.51$. 


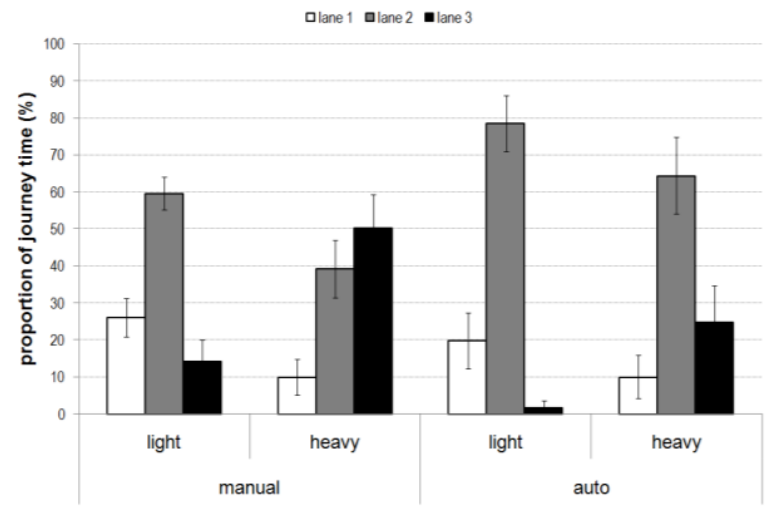

Figure 2. Lane occupation in the three available driving lanes (error bars show 95\% C.I.)

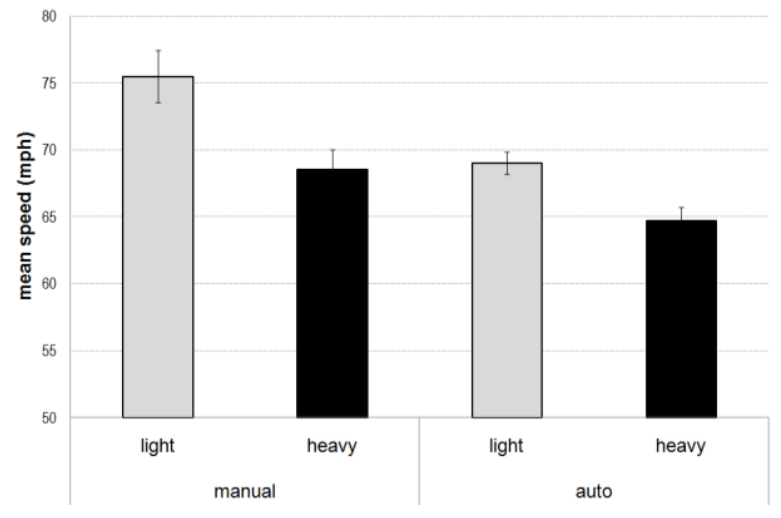

Figure 3. Mean speed (error bars show $95 \%$ C.I.)

Naturally, there was a reduction in mean speed from light to heavy traffic; $F(1,48)=168$, $p<.00001, \eta^{2}=.78$. However, there was also a significant interaction such that the reduction in mean speed from light to heavy traffic was less pronounced for automated control than it was for manual driving; $\mathrm{F}(1,48)=11.0, p=.002, \eta^{2}=.19$ (Figure 3). This observation is in line with Hall and Chin's (2005) real world demonstration that vehicle automation harmonises vehicle flow. This is even more relevant given that, even in busy traffic, participants would have been able to at least match the observed mean manual driving mean speed, but through their use of the automated system, consciously or subconsciously chose not to do so.

The margin of longitudinal safety was assessed with respect to time-exposed-time-to-collision (TETTC), defined as the percentage of journey time in which time-to-collision was less than 20s. There was evidence to suggest that automated driving appeared to improve safety margins as TETTC decreased from manual $(8.26 \%)$ to fully-automated driving $(5.82 \%) ; \mathrm{F}(1,48)=14.8$, $p<.001, \eta^{2}=.24$. However, those improved margins tended to be only limited to light traffic conditions, a significant interaction of System Type and Traffic being observed suggesting that in busy traffic the automated system handled longitudinal control not dissimilarly to manual driving; $\mathrm{F}(1,48)=4.65, p=.031, \eta^{2}=.22$.

\section{Driver physiology}

There was a main effect of Driver Type on heart rate, such that the number of beats per minute (bpm) decreased from $82.1 \mathrm{bpm}$ in manual driving conditions to $75.6 \mathrm{bpm}$ when the task was fully automated; $\mathrm{F}(1,48)=13.6, p=.002, \eta^{2}=.23$ There was also evidence to suggest that automation increased fatigue, with PERCLOS (Wierwille, Ellsworth, Wreggit, Fairbanks and Kirn, 1994) significantly increasing from 0.018 in manual driving to 0.038 in the corresponding fully-automated condition; $\mathrm{F}(1,48)=6.10, p=.018, \eta^{2}=.13$. There was also a significant interaction of Drive Type and Traffic Density (Figure 4) to suggest that heavy traffic conditions did mitigate reduced arousal levels in automated driving. More fatigue was evident than demonstrated in manual driving, which in contrast appeared unaffected by Traffic Density. 


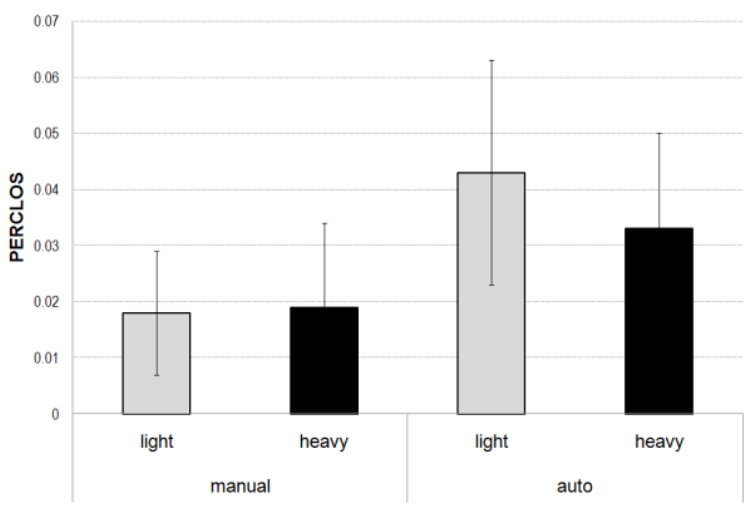

Figure 4. Proportion of over-pupil eye-closures (error bars show $95 \%$ C.I.)

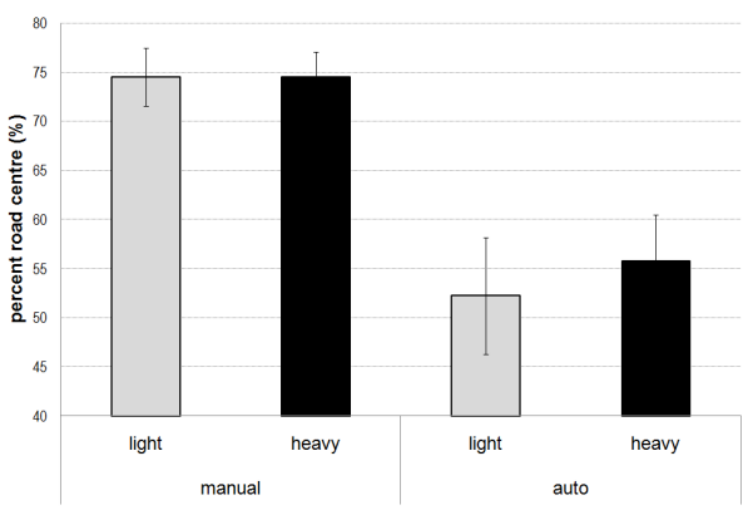

Figure 5. Percent road centre (error bars show $95 \%$ C.I.)

\section{Secondary tasks}

Drivers did show an inclination to become more heavily engaged in both visually demanding and undemanding in-vehicle entertainment. Use of the radio significantly increased from $41.1 \%$ in manual driving to $54.1 \%$ when experiencing full vehicle automation; $\mathrm{F}(1,48)=8.59, p=.018$, $\eta^{2}=.13$. There was a also dramatic rise in drivers playing their chosen DVD in automated conditions $(32.5 \%)$ as opposed to manual control $(2.6 \%) ; \mathrm{F}(1,48)=22.3, p<.001, \eta^{2}=.32$.

Such partiality to the entertaining tasks may have contributed the shorter duration that drivers spent fixated within the road centre area when under automated vehicle control (Figure 5). Percent Road Centre (PRC), the percentage of gaze data points that fell within the road centre area, a $6^{\circ}$ circular region located around the driver's most frequent fixation location. PRC decreased significantly from $74.5 \%$ when driving manually to $54.0 \%$ when automated, suggesting a reduction in visual attention to the primary driving task and an increase to those associated with the entertaining secondary tasks; $\mathrm{F}(1,48)=64.9, p<.0001, \eta^{2}=.63$.

Figure 5 also shows the observed interaction of Drive Type and Traffic Density; $F(1,48)=4.41$, $p=.042, \eta^{2}=.11$. Manual drivers demonstrated the same visual attention to the roadway regardless of the surrounding traffic. However, drivers experiencing automation, whilst having a longer period focussed away from the road, were gripped to demonstrate additional attention in heavy traffic, where increased vigilance in automated system monitoring is more necessary.

\section{CONCLUSIONS}

The hypothesis that vehicle automation leads to more efficient traffic flow was unproven. Whilst mean speed did reduce under automation, this was a result of drivers' reluctance to intervene, limiting their manual lane changing behaviour and becoming held up by traffic. To overtake in the fully-automated condition, drivers would have had to disengage the system, manually perform the lane change/overtaking manoeuvre, return to the lane of choice and re-engage the system. Clearly, this was not undertaken even though it resulted in a longer journey at reduced mean speed. 
Along with the efficiency of fully-automated driving, any evaluations on its safety implications also need to be tempered by an acknowledgement of the major role that the specific system design plays in its behaviour. That said, there was a suggestion that automation does contribute towards a safer driving environment, demonstrated by the significantly shorter period exposed to low time-to-collision. Such a finding concurs with previous work, particularly focused on ACC (e.g. Stanton and Young, 1998; Young and Stanton, 2004). However, these improved margins are limited to conditions of light traffic conditions. A significant interaction of System Type and Traffic Density was observed suggesting that in heavy traffic automation handles longitudinal control in a similar fashion to that exhibited by drivers controlling the vehicle manually.

The final hypothesis was that vehicle automation creates a less stressful driving environment. Drivers experiencing automation did display a significantly reduced heart rate when compared their manual driving. However, to conclude that the automated driving environment was therefore less stressful must to be moderated by the fact that heart rate can also fluctuate for a number of other reasons in driving such as the strains of physical (see Stoynev and Minkova, 1997) or mental effort (see Brookhuis and de Waard, 2010). Nevertheless, automated driving is undeniably free of the rigours of physical and mental effort associated with manual driving and any suggestion of such reductions do result in a lower pulse rate are likely to be beneficial, supporting Stanton and Young's (2005) assertion that the reduction in workload associated with the automation of the driving task also has the potential to significantly alleviate driver stress.

Stress can arise, however, from fatigue in driving (Desmond and Matthews, 2009). Using eyetracking data to infer driver fatigue through PERCLOS, there was evidence to suggest that, although mitigated by the demands of busy traffic, vehicle automation reduces arousal and increases fatigue. Manual driving had no impact on fatigue, regardless of the prevailing traffic conditions, as result that concurs with the observations of Saxby et al. (2008).

The opportunity for vehicle automation to freeing up attentional resources was most definitely exploited by drivers, who showed strong propensity to become involved by in-vehicle entertainment, when under automated rather than manual control. This effect was observed both for the more traditional, non-visually demanding use of the radio, and even more strongly for the less familiar (and more visually conflicting) opportunity to watch a personally-selected DVD whilst driving. Such inclinations to embrace the in-vehicle tasks was coupled with significantly longer durations of visual attention away from the road, indicated by the very strong reduction in Percent Road Centre from manual to automated driving. Clearly, participants were willing to compromise their requirements to continually monitor the automated system, exhibiting much confidence in its ability.

However, there was also an interesting interaction of Drive Type and Traffic Density for PRC. Manual drivers showed the same visual attention to the roadway regardless of the traffic conditions. Automated drivers, on the other hand, whilst spending a significantly longer period fixating away the road, did demonstrate more visual attention as traffic conditions became heavier. This suggests that even though the third hypothesis was proven, its negative impacts can be minimised to some extent by vehicle automation that is both reliable and ergonomically welldesigned. 


\section{REFERENCES}

Bainbridge, L. (1983). Ironies of automation. Automatica, 19(6), pp. 775-779.

Brookhuis, K.A. \& de Waard, D. (2010). Monitoring drivers' mental workload in driving simulators using physiological measures. Accident Analysis and Prevention, 42(3), pp. 989903.

Hall, R. \& Chin, C. (2005). Vehicle sorting for platoon formation: impacts on highway entry and throughput. Transportation Research Part C: Emerging Technologies, 13(5-6), pp. 405-420.

Ioannou, P., Xu, Z., Eckert, S., Clemons, D. and Sieja, T. (1993). Intelligent Cruise Control: theory and experiment. Proceedings of the $32^{\text {nd }}$ IEEE Conference on Decision and Control, San Antonio, Texas, December 1993, pp. 1885-1890.

Nilsson, L. (1995). Safety effects of adaptive cruise controls in critical traffic situations. Proceedings of the Second World Congress on Intelligent Transport Systems, Yokohama, Vol. 3, pp. 1254-1259.

Norman, D.A. (2007). The design of future things. New York: Basic Books.

Parasuraman, R., Sheridan, T. B. \& Wickens, C. D. (2000). A model of types and levels of human interaction with automation. IEEE Transactions on Systems, Man, and Cybernetics Part A: Systems and Humans, 30(3), pp. 286-297.

Saxby, D.J., Matthews, G., Hitchcock, E.M., Warm, J.S., Funke, G.J. \& Gantzer, T. (2008). Effects of active and passive fatigue on performance using a driving simulator. Proceedings of the Human Factors and Ergonomics Society $51^{\text {st }}$ Annual Meeting, pp. 1237-1241. Santa Monica, CA: Human Factors and Ergonomics Society.

Sharp, R. S., Casanova, D. \& Symonds, P. (2000). A mathematical model for driver steering control with design, tuning and performance results. Vehicle System Dynamics, 33(5), pp. 289-326.

Stanton, N.A. \& Marsden, P. (1996). From Fly-By-Wire to Drive-By-Wire: safety implications of automation in vehicles. Safety Science, 24(1), pp. 35-49.

Stanton, N.A. \& Young, M.S. (2005). Driver behaviour with adaptive cruise control. Ergonomics, 48(10), pp. 1294-1313.

Stoynev, A.G. \& Minkova, N.K. (1997). Circadian rhythms of arterial pressure, heart rate and oral temperature in truck drivers. Occupational Medicine, 47(3), pp. 151-154.

Wiener, E. L. \& Curry, R. E. (1980). Flight-deck automation: promises and problems. Ergonomics, 23(10), pp. 995-1011.

Young, M.S. \& Stanton, N.A. (2007). Back to the future: brake reaction times for manual and automated vehicles. Ergonomics, 50(1), pp. 46-58. 